\title{
Glioma stem cells and the occurrence of tumor blood vessels
}

\author{
Yude Zhu , Qiang Huang \\ The Third Affiliated Hospital of Inner Mongolia Medical University, Hohhot, China
}

Received: March 1, 2015

DOI: $10.14725 /$ dcc.v2n2p12

\author{
Accepted: April 2, 2015 \\ Online Published: June 15, 2015 \\ URL: http://dx.doi.org/10.14725/dcc.v2n2p12
}

\begin{abstract}
Epithelial glioma is the most common brain cancer, accounting for $35.26 \%-60.69 \%$ of intracranial tumors with an average of $44.69 \%$, and it remains the greatest challenge in the field of neurosurgery. The median survival time of patients with advanced glioma is only 12 to 18 months due to the characteristics of high aggression, and the therapeutic effect was poor though surgery, chemotherapy, and targeted drug therapy being treated. Because of the presence of heterogeneity and the differentiation disorder, only a small number of glioma cells are the source of tumor growth and metastasis, which are highly resistant to traditional treatments. They are deemed as the "seed" tumor cells as they could get rid of the effect of the treatment and reconstruct the organization of tumor. They are also termed as brain tumor stem cell (BTSC) or glioma stem cells (GSCs) since neural stem cells share similar features with them. Recent data reveal that they are directly related with invasion, angiogenesis, tolerance, chemotherapy, recurrence of glioma. Based on the research result by the team, the paper elaborates the characteristics of GSCs and the relationship with the tumor angiogenesis.
\end{abstract}

Key Words: Glioma stem cells, Tumor blood vessels

Epithelial glioma is the most common malignant tumor of the brain tumors, ${ }^{[1]}$ accounting for $35.26 \%$ to $60.69 \%$ of intracranial tumors, with an average of $44.69 \%$, and its corresponding treatment remains a challenge in the field of neurosurgery. Despite the use of the best available multimodal therapeutic approaches, such as surgery, radiotherapy and chemotherapy, and targeted drug therapy, it still offers a dismal patient prognosis due to highly aggression in high grade gliomas, and the average survival period is only 12 to 18 months. ${ }^{[2,3]}$ In fact, only a minority of cancer cells, similar to seed cells of tumor, is the source of tumor growth and metastasis due to heterogeneity and differentiation disorder, which imposes conventional therapy resistance and escape the destruction for the construction of tumor. They are known as brain tumor stem cell (BTSC) or glioma stem cells (GSCs) due to similar characteristics with neural stem cells. Recent data shows that ${ }^{[4-8]}$ it has a direct relationship with the invasion, angiogenesis, tolerance, chemotherapy, recurrence and so on. The discovery of GCSs provides a new path for the study and treatment of gliomas. Our paper mainly focuses on GSCs's own characteristics and its relationship with the tumor angiogenesis, and the research results are introduced as follows.

\section{The characteristics of glioma stem cells}

\subsection{Glioma stem cell therapy}

Stem cell (SC) refers to the cell that possesses two properties of self-renewal and multi-differentiation. Usually, it is divided into three categories according to differentiation caChina.

*Correspondence: Yude Zhu; E-mail: hq1936@163.com; Address: The Third Affiliated Hospital of Inner Mongolia Medical University, Hohhot, 
pacity. (1) Embyonic stem cell (ESC), also known as totipotent stem cell (TSC), can differentiate into any specialized cell types. (2) Pluripotential stem cell, with multiple differentiation potential, has the potential to differentiate into a variety of tissue cells, meanwhile it losses the ability to develop into a complete individual. Among them, bone marrow derived from stem cell (BMSC) and hematopoietic stem cell (HSC) are the representative types. (3) Adult stem cells (ASC), also known as unipotent stem cell (USC), progenitor cells or precursor cells, and cancer stem cells (cancer stem cell, CSC), belongs to the category of adult stem cells.

There are many names for cancer stem cells in the literature, such as pre-cancerous stem cells (pCSCs), side population cell (SP cell), cancer stem cells (CSC), stem-like cells, tumor initiating cells (TIC) and so on. The doubt whether they the same tumor initiating cells, or GSC is not a single group remains to be unveiled. Therefore, American Association for Cancer Research (AACR) normalized the definition: cancer stem cells refers to the tumor cells that possess the capacity of self-renewing and heterogeneous production.

The concept of tumor stem cells was first raised in the study of hematologic malignancies, indicating that only a small group of cells possess the ability to infinite proliferation rather than the whole cells. Animal experiments demonstrate that immature leukemic cells in the CD34+/CD38- stimulate the formation of tumor in mice with immunodeficiency, while the mature leukemic cells in the CD34+/CD38+ cannot form tumor. ${ }^{[9-11]}$ Tumor stem cells has been generally accepted when they are successively identified in the tumor tissues of leukemia, breast cancer, brain cancer, lung cancer, skin cancer, liver cancer, pancreatic cancer and colorectal cancer, along with the development of in-depth research.

\subsection{Origin of glioma stem cell}

In 1920s, Bailey speculated that cells of origin of glioma derived from normal cells of the same name in 1920s as he found the same morphology of astrocytoma cells as astrocyte, and oligodendrocyte cells as oligodendrocytes via microscope observation. When these tumors become more malignant, the cancer cells show the characteristics of low differentiation of precursor cells so that the malignant astrocytoma is also called astrocytoma. However, the discovery of neural stem cells undermines the views above. ${ }^{[12]}$ CSC is regarded as the "seed" cell of tumor growth, but it merely emphasizes that only few cells in the tumor tissues possess the characteristics of infinite proliferation and parental differentiation, while other cells have finite proliferation capacity. It is concluded that the origin of CSCs is related to the stem cell. ${ }^{[13,14]}$ Meanwhile, tumor tissue is from an undifferentiated stem cell like CSCs or tumor initiating cells, which constitute one or several subpopulations of the tumor, and plays an important role in the tumor's initial launch. ${ }^{[14]}$
The origin model is shown in Figure 1.
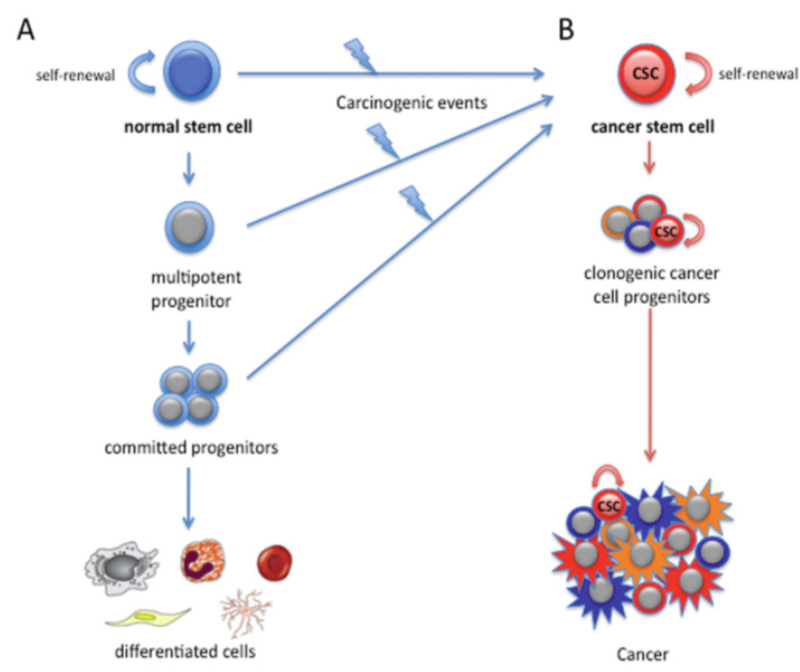

Figure 1: Origin and development patterns of cancer stem cells. (A) Normal tissue stem cell may generate stable stem cell through process of self-renewal, or produce progeny which could not renew themselves but can differentiate into all mature cell types in their tissues. The progeny will differentiate into specific (committed) stem cells and finally into mature cells. Genetic or epigenetic mutations may potentially occur in normal stem cells due to its self-renewal capacity. (B) Tumor and its origin have similar organizational structure. Tumor stem cells generate new tumor stem cells or progeny and eventually differentiate into the cells in the tumor tissues by self-renewal. Although there is no direct evidence for the origin of cancer stem cells, it is possible that he derives from normal stem cells or acquires the ability to renew themselves.

Cellular malignant transformation gradually results from multi-gene mutation in cells. In theory, the differentiated cells could not complete the accumulative process of these multiple variants due to its short life cycle. The biological characteristics of stem cells, such as self-renewal and long-term survival and infinite proliferation, enable the possibility for multiple mutation accumulation. ${ }^{[4]}$ However, in 2006, Takahashi, a Japanese scholar transplanted OCT3/4, Sox 2 and c-myc and KLF4, 4 kinds of transcription factor gene into the mouse fibroblast cells successfully, which induced the dedifferentiation and exhibited stem cell properties when he conducted researches about induced Pluripotent stem cells (iPS). ${ }^{[15]}$ Academician Ceng Yixin proposed a new tumor cells origin theory ${ }^{[16]}$ that ordinary tumor cells can also evolve into GSCs induced by chromosomal instability, which was then known as stem cells like tumor derived cancer cells (SLCC). Previously, the origin of TSCs remained controversial, during which Chen proposed the concept of pre CSCs. ${ }^{[17]}$ They isolated a new set of cells, which 
were somewhat similar to CSCs, from the tumor tissue. The new cells turned to different outcomes when induced by different cellular signaling. Some grow into cancer cells or cancer stem cells, while some also maintain active state or removed from the body's immune system. The researchers called these unusual cells CSCs. The theory of pre CSCs is an important supplement to CSCs theory, which is the source of the CSCs. Though more researches are required to demonstrate the existence of pre CSCs, it has vital implications for the occurrence and development of CSCs model.

\subsection{Surface marker of cancer stem cells}

1.3.1 The essential factors for identification of CSCs include the "tumor spheres", oncogenicity, multi potential differentiation, single cell cloning and stem cell marker, among which stem cell marker serves as the most frequently used method by researchers

CD133, also known as Prominin-1, is a transmembrane protein. It is recognized as a stem cell marker due to its strong positive expression in juvenile cell but the expression decreased or even disappeared along with cell differentiation and maturation process. Usually, monoclonal antibodies are used to detect the presence and content of the target protein. All the current CD133 antibodies, such as AC133, ac141, 293c verify the existence of the protein by detecting glycosylation site on the surface. The precise definition of CD133 as a GSCs marker by immunohistochemistry and cell chemistry should be: CD133 glycosylation of the positive glioma cells is GSCs. It is possible that CD133 changes in the differentiation process, that is, the glycosylation epitope of CD133 protein is gradually reduced, but not the whole protein. ${ }^{[21]}$ The growth pattern of CD133+ and GSCs is "spherical aggregates". [5] We may find the available GaIC, GFAP and MAP2 cells expressed when induced to differentiation, indicating that they are primitive. The single isolated GSCs potentially clones and gradually forms a "spherical aggregates" for its self-renewal ability. Also, it shows strong oncogenicity of GSCs when transplanted into tumor cell though under minimal number.

Recent research confirms that a small group of CD133 + glioma cells share the characteristics of stem cells, which undermines the role of CD133 as GSCs test "standard". ${ }^{22-26] ~ C D 133+~ c e l l s ~ a r e ~ i s o l a t e d ~ f r o m ~ g l i o m a ~ b y ~}$ flow cytometry or immunomagnetic beads and the remaining CD133- proceeds into tumor spheres. CD133 + tumor cells could not be detected in some primary gliomas, and $0.5 \% 2 \%$ CD133 tumor cells could aggregate into spherical cells, but a large number of glioma cells did not possess the growth characteristics of stem cells. The CD133 glioma cells which were clustered into spheres show no expression of related neuro-biology marker in the natural state, but the expression of GaIC, GFAP and MAP2 are obtained after differentiation induction. We made some discoveries when aggregation of CD133 glioma cells had been diluted into a single cell for culture that $16.9 \%-53.8 \%$ could clone and re-formed tumor sphere, showing no significant differences from CD133GSCs. CD133- glioma cells produces a lot of adherent glioma cells in the formation of the tumor sphere, but they lost previous ability to clone when the adherent cells were diluted into single cells again. A part of CD133- glioma cells with the capacities of aggregate sphere, pluripotent and self-renewing are found in animal models to carry strong oncogenicity. Only 105-106 cells are essential for the formation of tumor in NOD/SCID mice, showing similar phenotype to the primary tumors. In addition, there is no significant difference from CD133+ GSCs in forming tumor in view of the morphology, the size and the formation time of tumor. On the contrary, ordinary glioma cells do not have oncogenicity.

It is worth noting that CD133 glioma cells, accompanied by these features above, show expression of stem cell marker Nestin. ${ }^{[27]}$ Lottaz et al. ${ }^{[28]}$ confirmed that little part of CD133- glioma cells share similar transcript with NSCs, especially similar pathways in anti-apoptosis, but most of the CD133- glioma cells do not possess such similar molecular mechanisms. CD133-GSCs also has the characteristic of stem cells, which contradicts that theory that CD133+ is the unique origin of glioma, indicating that CD133 is not a reliable tumor stem cell marker. The joint detection technology is widely applied and it allows more markers to be detected, such as CD133, Nestin.

\subsubsection{Others}

A2B5 is a neural cell surface antigen predominantly expressed in NSCs, whose its positive cells have the potential to differentiate into astrocytes and microglia. The isolated A2B5+ tumor cell enables the formation of a "tumor" no matter how CD133 is expressed, and it carries oncogenicity since the sum reaches 103 . Yet, it is impossible for A2B5 $-{ }^{[29]}$

Some data indicate that A2B5 tumor cells occupies a large proportion of the tumor component, ${ }^{[30]}$ which contradicts to the idea that CSCs is only a small population of tumor cells. GSCs may be contained in a portion of the population of A2B5 tumor cells. SoX2 (homoebox transcription factor SRY-related 2) is a transcription factor that maintains the versatility of stem cells and serves as a marker of NSCs, and its expression is decreased along with the process of differentiation and maturation, which is similar to CD133. It is also able to express ${ }^{[31]}$ in the tumor spheres that were sorted out from the gliomas. Annovazzi et al. ${ }^{[33]}$ found that SoX2 + glioma cells had the ability to inhibit differentiation, unlimited proliferation and oncogenicity. However, the practical employment of GSCs for SoX2 separation and its reliability is rarely reported so that more available researches are advisable. 
Side population cell (SP cell) refers to a group of low staining cells when stained with Hoechst33342 dye. Current literatures mainly focus on the correlation between CD133+ GSCs and SP cell, which impeded the studies on whether CD133GSCs and CD133GSCs could be contained simultaneously in SP. Not all SP cells are present in the "tumor spheres". Non-SP cells possess stronger capacities of selfrenew and oncogenicity, and they are prone to transform into SP cells, suggesting that GSCs is a part in non-SP cells. Though there is not enough evidence to show that these markers contain different subtypes of GSCs, it lays a solid foundation for the discovery of more specific markers and targeted therapy of GSCs when combined with Nestin.

\section{Cancer stem cells and tumor microenvi- ronment}

A large number of studies corroborates that the stability of normal tissue microenvironment is an important condition for maintaining normal cell proliferation, differentiation, metabolism and functional activities. Although early cancer researches often focus on the tumor cells themselves, pay attention to the tumor cell gene mutation, growth and proliferation and signal pathways of change, the theory that tumor micro environment serves as the necessary structure and functional unit to protect and sustain tumor occurrence, metastasis and recurrence gains growing support as the research further develops. Tumor is more likely to be considered as a disease of stem cells to date. ${ }^{[34,35]}$ Cancer stem cells rely on a specific microenvironment to maintain the stem cell characteristics. Tumor microenvironment is a complicated system, consisting of a number of stromal cells, such as fiber cells, immune and inflammatory cells, fat cells, glial cells, and smooth muscle cells and vascular cells. ${ }^{[36,37]}$ Although the mechanisms of cancer stem cell microenvironment formation have not been fully unveiled, the role of various components in the microenvironment in the regulation of tumor stem cells has been demonstrated. ${ }^{[38-40]}$

\subsection{Hypoxia}

Hypoxia is regarded as an independent prognostic factor for tumor progression, resistance to conventional treatment and decreased tumor free survival. Hypoxia is widespread in solid tumors, and accelerates tumor malignant transformation by inducing biological behavior changes, such as inhibition of cell proliferation, enhance expression of the resistance gene, selectivity of anti- apoptosis, promotion of tumor invasion and metastasis, down-regulation of expression of DNA repair genes and increased genetic instability $^{[39,40]}$ Most studies have indicated that hypoxia has the ability to regulate the differentiation of cancer cells, which can enhance the malignant potential tumors by promoting

Published by New Century Science Press the formation of cancer stem cells and epigenetic modification. ${ }^{[41,42]}$

Kim et al ${ }^{[43]}$ certified the existence of hypoxic tumor microenvironment via hypoxic markers, such as EF5 or endogenous molecules indications (such as hypoxia inducible factor 1 alpha and carbonic anhydrase 9). Hypoxia is crucial to maintain the biological characteristics of GSC. Hypoxic microenvironment enables the interaction between cells and the maintenance of tumor stem cells in an undifferentiated state. The undifferentiated stem cells allows tumor cells to remain permanent self-renewal and repair capacity, to generate related genetic and epigenetic changes in order to facilitate tumor cells into malignancy.

Hypoxia-induced signal pathway is mainly under the regulation of hypoxia inducible factor-1 (HIF-1). ${ }^{[4]} \mathrm{HIF}-1$ is the chief regulatory factor in maintaining oxygen homeostasis. Research shows that, compared with normal non ischemic tissue, enhanced expression of HIF-1 and protein could be detected in solid tumors by which the significant correlation with low survival rate could not be denied. ${ }^{[45]}$ Jianchun Zong et al. ${ }^{[46]}$ confirmed that the prognosis of breast cancers was much associated with HIF- $1 \alpha$ mRNA and the protein level. Further study demonstrated that HIF- $1 \alpha$ protein nuclear accumulation was positively correlated with low differentiation of pancreatic cancer cells and expression ${ }^{[47]}$, that is, higher expression equals to worse prognosis. Acker et al. ${ }^{[48]}$ proved that the expression of HIF-2 can hamper the growth of glioma cells, which reflects the differences in the regulation of HIF relates to the types of tumor cells and the microenvironment. Recent studies have shown that compared with HIF-1, HIF-2 in glioma stem cells, the expression of HIF-1 and HIF-2, silent HIF- $1 \alpha$ and HIF- $2 \alpha$ is higher in glioma stem cell subsets, and they inhibit the formation of neurospheres and effect on glioma stem cell survival. ${ }^{[49,50]}$ In the study of human glioblastoma, Pietras et $\mathrm{al}^{[51]}$ found that HIF-2 $\alpha$ firstly expressed in the immature nerve of the spinal neuroblastoma, while maintaining the undifferentiated state of neuroblastoma. All the studies above suggest that the HIF pathway plays a critical role in the maintenance of cancer stem cells in undifferentiated phenotype.

The formation of fence-shaped structure around tumor necrosis focus is a significant pathologic feature that distinguishes GBM from low grade gliomas. Fence-shaped structure is a result of tumor cell proliferation and insufficient nutrition offered to angiogenesis. The structure extents out constantly, leading to the formation of coagulative necrosis. Recent studies have also verified that the high expression of glioblastoma cells of stem cell related genes distributed around the hypoxic tumor necrosis, and HIF-1 $\alpha$ and HIF- $2 \alpha$ are positively expressed accordingly. ${ }^{[52]}$ But other studies figured out the presence of brain tumor stem cells in surrounding areas of blood vessels. ${ }^{[53,54]}$ Pietras et al. ${ }^{[51]}$ also discovered some immature neural crest cells scattering 
in the blood vessel surrounding area. The latter study underlies that the cancer stem cell nests are not necessarily the hypoxic environment. However, vitro co-culture experiments demonstrated the ability of endothelial cells to promote cancer stem cell self-renewal and growth, showing that endothelial cells can directly regulate cancer stem cell renew independently, other than in the combination of blood circulation or other vascular functions. Therefore, it is included that there is a close relationship between tumor stem cells and vascular endothelial cells in hypoxia areas. According to the current study, tumor stem cells may exist in two different microenvironments, ${ }^{[55]}$ that is, away from the vascular function of hypoxic microenvironment or in hypoxia or nonhypoxic microenvironment around blood vessels. There are at least three possible mechanisms for hypoxic regulation of cancer stem cell generation and differentiation: (1) hypoxia inhibits cancer stem cell differentiation directly; (2) hypoxia inhibits the differentiation of interstitial cells in the microenvironment and maintain their undifferentiated state; (3) hypoxia encourages tumor stem cells to locate near endothelial cells, or hypoxia or non-hypoxic microenvironment around blood vessels through the regulation of paracrine factors of endothelial cells.

\subsection{Tumor-associated fibroblasts (TAFs)}

TAFs are heterogenous cell populations, which can be derived from a variety of cells including quiescent fibroblasts, epithelial cells, endothelial cells and mesenchymal stem cells. In vivo and vitro experiments, fibroblasts play a crucial role in tumor development under tumor microenvironment, instead of providing support passively. In that case, some researchers have proposed a target to TAFs is expected to become the new direction of cancer therapy. Recent studies found that, ${ }^{[57-59]}$ activation of fibroblasts from adjacent tumors was active in regulating the homeostasis of the tumor microenvironment, even determined the fate of the tumor cells. TAFs usually results from epithelial - mesenchymal transition, endothelial-mesenchymal transition, differentiation of mesenchymal stem cells and quiescent fibroblasts. Since the cells have both some characteristics of fibroblasts and myofibroblasts, it is also called myofibroblasts or tumor associated fibroblasts (TAFs) or cancer associated fibroblasts (CAFs), whose features are distinctive from those of fiber cells within inflammation and trauma environment. TAFs can promote tumor angiogenesis by secretion of Pro angiogenic factors and MMPs, regulate the immune response by secretion of inflammatory chemokines, or promote the proliferation and invasion by direct action on tumor cells. Additionally, TAFs also secrete factormediated tumor cells for tolerance of anti VEGF therapy. In the meantime, it produces a variety of cytokines, growth factors, protease and extracellular matrix components when its functions of proliferation, motility, secretion increase significantly than normal fibroblast, which expedites epithelial cell differentiation, proliferation, invasion on one hand, and affects other components of the host, and eventually stimulates tumor occurrence and development on the other hand.

\subsection{Tumor-associated macrophages(TAMs)}

Chemokines and their receptors mediate the recruitment of macrophages of the immune system to the tumor microenvironment and the interaction between macrophages and tumor cells contribute to the formation of tumor associated macrophages. The activated TAM accelerates tumor associated angiogenesis, invasion, infiltration and metastasis by secreting a variety of cytokines and chemotaxis factors. The process is also an important part of immune regulation. Nuclear transcription factor- $\kappa \mathrm{B}(\mathrm{NF}-\kappa \mathrm{B})$, Toll like receptor (TLR) may potentially facilitate growth and proliferation of tumor cells via activation of signaling pathways. The functions above are affected by the tumor microenvironment of TAM. Past studies on multiple human solid tumors and hematological malignancies raised the possibility that TAM might be associated with a poor prognosis. ${ }^{[60]}$ Macrophages are important effector cells and antigen presenting cells in humoral and cellular immunity. TAM is involved in the occurrence and development of tumor, and the number of TAM invasion is closely related to the prognosis of tumor. As a consequence, the intervention of various biological behaviors during the evolution of TAM is a hot spot in the research of tumor immunity. The homing ability of TAM to the tumor tissue offers a new thread for molecular targeted therapy of TAM in treating and diagnosing tumors. It is an effective means of anti-tumor therapy. The study showed that the invasiveness of the tumor was directly related to the number of TAMs nearby. ${ }^{[61]}$ Compared with normal macrophages, TAMs is characterized by changes in gene expression, secretion of prostaglandin, the ability to activate oxygen metabolism products enhancement and the inhibition of lymphocytes. Moreover, the expression of VEGF, PDGF, IL-8 in TAMs contributes to the formation of peripheral blood vessels and lymphatic vessels. However, the expression of costimulatory factor (B7-1/B7-2) in TAMs activates $\mathrm{T}$ cells to be involved in anti-tumor immune response by direct contact with lymphocyte. It could be concluded that TAMs impose dual effects on tumor development, while its mechanism needs to be further explored. ${ }^{[62]}$

\subsection{Tumor-associated endothelial cell}

Tumor-associated endothelial cell ascend from the host mature endothelial cells, and the latter divides, proliferates and establishes new blood vessels in tumor microenvironment. Tumor angiogenesis is a complex process, including vascular endothelial basement membrane dissolution, endothelial cells migration to the tumor, and endothelial cells proliferation in the front migration, channelization of endothelial 
cells pipeline, branches form part of the vascular ring, and formation of a new basement membrane and other steps. On one hand, the phenotype of tumor-associated endothelial cells is distinctive as a result of different tumor types. On the other hand, affected by the tumor microenvironment, microvascular endothelial cells and endothelial cells adjacent to tumor inoculation site itself share similar characteristics when the same kind of tumor cells were inoculated in corresponding parts. ${ }^{[63,64]}$ Compared with the normal vascular endothelial cells, endothelial cell in the tumor microenvironment is characterized by immature, rapidly dividing in the activation state, and it plays an important role in the rapid proliferation of vascular tumors, tumor rapid development, invasion and metastasis. In addition, the functions of other host cells such as T cells, B lymphocytes, natural killer cells and neutrophil cells, nerve cells, glial cells, pigment cells in the tumor host interface microenvironment could not be ignored.

\subsection{Extracellular matrix(ECM)}

Extracellular matrix is a generic term for all solid phase proteins located outside of the cell, mainly including collagen protein, glycoprotein, protein and elastin. ECM is present in the epithelial basement membrane (basement membranes, $\mathrm{BM}$ ) in the form of basement membrane. Recent studies found that ${ }^{[65]}$ ECM was not only responsible for the support and nourishing cells, providing survival environment, but also involved in cellular physiological and pathological processes, including tumor cell proliferation, differentiation, adhesion, migration, and other important process as a solid phase cell or neural tube signal transduction system due to the unite between a variety of integrin or integrin receptor cell and ECM at the cell surface.

\subsection{Tumor-secreted factor}

The interaction among the components of the tumor microenvironment functions is involved with elaborate and complex signal transduction network, among which cytokine stands out the most. It is mainly secreted by cells of the immune system, such as interferon, interleukin, set colony stimulating factor, tumor necrosis factor, growth factor, a family of chemotactic factor and so on. Cytokine imposes dual effect on tumor, on the one hand, it can kill tumor cells and inhibit tumor growth; on the other hand, it may promote tumor growth, even play a key role in the occurrence and development of some tumors. ${ }^{[66]}$ Cell adhesion molecules serve as a mediator at the cell surface for the combination of between cell and cell or cell and matrix, which is of significance in tumor invasion and metastasis. ${ }^{[67]}$ Common adhesion factors include integrin, calcium, immunoglobulin superfamily, and so on. Others also include motion related molecules (such as autocrine mobile factor, mobile stimulating factor, scatter factor), tissue dissolving enzymes (fibrinolytic enzyme, cathepsin, matrix metalloproteinase), hormones, neurotransmitters (such as growth hormone, acetylcholine, adrenaline) are essential for tumor microenvironment. ${ }^{[68,69]}$

\section{Tumor stem cells and angiogenesis}

\subsection{Regulatory mechanism of angiogenesis}

Previous studies view that tumor angiogenesis undergo the following steps: ${ }^{[70]}$ (1) endothelial cell activation and vascular endothelial basement membrane degradation; (2) proliferation of endothelial cells and migration to the tumor tissues; (3) endothelial cell develops into cell line- and lumenshaped structure. In addition to the interaction between tumor cells, normal endothelial cells and extracellular matrix interactions during the whole process, other cell factors, such as pro-angiogenic factor and anti-angiogenic factor, are involved in the process. Pro-angiogenic factor expression is higher than its counterpart when the balance between the two breaks, result in the formation of tumor vessels. ${ }^{[71]}$ Therefore, any abnormal regulatory factors that lead to endothelial cell proliferation, differentiation, migration and angiogenesis may affect angiogenesis.

The most identified angiogenic factors include vascular endothelial growth factor (VEGF), platelet-derived growth factor (PDGF), transfoming growth factor (TGF), basic fibroblast growth factor (bFGF), epidermal growth factor (EGF), and insulin-like growth factor (IGF). Hypoxia response is a key step in the start-up and development of tumor angiogenesis. Hypoxia inducible factor -1 (HIF-1) gene is a critical regulator of tumor microenvironment, which plays a central role in promoting tumor angiogenesis and tumor invasion. ${ }^{[72]}$ The biological characteristics of glioma adhere to general rule of tumor development. That is, higher nutrient demand by the glioma cells for the continuous growth drags tumor cells in a hypoxic state, which further activate HIF-1 to express, eventually lead to a series of gene activation, including vascular active factor, and their invasiveness enhanced. ${ }^{[73]}$ HIF-1 is capable of specific binding to $5 \mathrm{c}$ TACGTG-3c, a hypoxia induced gene promoter or enhancer of the hypoxia response element, and involved in transcription regulation of the corresponding target gene. It regulates expression of vascular endothelial growth factor (VEGF) at the genetic level. ${ }^{[74,75]}$

VEGF, one of the most identified factors, has a very close relationship with the invasion and migration ability of malignant glioma. CD133 + cells of GBM secreted high levels of vascular endothelial growth factor (VEGF), and promoted the migration of endothelial cell angiogenesis. CD133 + cells formed high vessel density of the tumor in the brain of immunodeficient mice. The application of VEGF monoclonal antibody bevacizumab in treating with 
CD133+ cells not only interdicts endothelial cell migration and tubular structures formation in vitro but also block tumor formation in vivo. ${ }^{[76]}$ BTSCs is an autocrine that secretes many chemokines and growth factors such as EGF, bFGF. Brekken et al ${ }^{[77]}$ found that VEGFR-2 played a major role in angiogenesis and vascular permeability induced by VEGF. KDR inhibitor alone may prevent the angiogenesis induced by VEGF and bFGF.

Basic fibroblast growth factor (bFGF), one of the 19 members of the FGF family, also known as FGF-2, is the first to be verified to promote the formation of blood vessels. Its bioactivity is mediated by high-affinity receptor (FGFR) and heparan sulphate proteoglycan as low affinity receptor. In addition, cysteine-rich FGF receptors and FGF binding proteins are bound up with secretion and bioactivity of bFGF. ${ }^{[78]}$ FGF-2 gene deficiency does not influence the growth and development of the mouse, but many tumor cell lines can produce FGF-2 in vitro, and the volume of FGF2 in the serum and urine was detected to be increased in many affected patients. Plenty of researches indicate that FGF-2 can promote cell mitosis and induce angiogenesis ${ }^{[79]}$ The up-regulated expression of FGF-2 in partly malignant tissue promotes sangiogenesis, increases the blood supply under the effect of carcinogenic factors, provides nutrition for the rapid proliferation of tumor cells, and then accelerates tumor growth. ${ }^{[80]}$ EL-Assal et al ${ }^{[81]}$ discovered that the expression level of FGF-2 was directly related to micro vessel density (MVD) and it played a significant role in fostering angiogenesis of tumors. FGF-2 could stimulate endothelial cells to secrete plasma urokinase type plasminogen activator (UPA) and collagenase and MMPs, which essentially leads to protein degradation around the basement membrane and extracellular matrix. FGF-2 has the capacity to drive proliferation and stimulation of endothelial cells, induce the expression of VEGF, imposing synergistic effect on VEGF. Acidic fibroblast growth factor (aFGF) and FGF2 were higher than the normal level in glioblastoma. ${ }^{[82]}$

At present, it is found that Ang1, Ang2 and their receptors play a major role in tumor angiogenesis. The predominant difference between Ang2 and Ang1 lies in the fact that quantity of cysteine in Ang2 at the junction of FL and CC is one fewer than those in Ang1, which constitutes their distinct biological functions. ${ }^{[83]}$ Ang1 binds to Tie-2 receptors, which triggers the interaction between vascular endothelial cells and perivascular cells, promoting vascular stability and maturation, and decreasing the function of vascular permeability. Ang2 is a natural antagonist of Ang1. Its combination with the Tie-2 receptor does not cause the phosphorylation of Ang1. Instead, it can hamper Ang1 to activate Tie2, reduce vascular stability so that Ang2 enables the blood vessels to re-enter the plasticity and unstable state. When VEGF appears, Ang2 hastens angiogenesis, vascular proliferation is significant, and cell membrane permeability increased obviously. Overexpression of Ang $2^{[84,85]}$ is directly related to angiogenesis, peritumoral brain edema and malig- nant progression of glioma.

The main difference between tumor angiogenesis process and normal angiogenesis lies in the following aspects: 1) the expression of KDR, a receptor of vascular endothelial growth factor (VEGF) enhanced; 2) vascular endothelial cell proliferation rate was higher than that of normal blood vessels; 3) the number of tumor vessels increased, distortion, uneven thickness and contain many abnormal the tumor vascular branches; 4) the lack of integrity of the basement membrane, incomplete endothelial cell coverage and large cell gap, resulting in high vascular permeability; 5) haemodynamics of tumor microvessel is abnormal, blood flow velocity in microcirculation increased. It is due to hypermetabolism and lack of smooth muscle in small artery wall. 6) unbalanced distribution of tumor blood vessels. Microvessel density around the tumor is greater than those in the central part. Vascular density of non-necrotic and cystic areas is higher than necrosis, cystic areas; 7) the tumor cells are involved in the constitution of vascular wall. Tumor angiogenesis is a complicated process, while the ultimate formation of lumen-like structure wherein endothelial cells as the center carries the real meaning of blood vessel. Recently, a novel endothelial cell-derived growth factor, named domain7 EGF-like, EGFL7, has been identified. Usually, expression of EGFL7 is reduced or disappeared in most normal adult tissues, but it shows a higher expression in vascular-rich tissues, such as lung, heart, uterus and ovary. In that case, EGF7 can be reactivated and involved in angiogenesis in physiological and pathological angiogenesis. ${ }^{[86]}$ Further studies indicated that, ${ }^{[87]}$ EGFL7 may modulate endothelial cell optimal migration path by determining the correct position of endothelial cells during vascular sprouting. In vascular sprouting process, neonatal endothelial cells constantly push its neighboring cells move forward due to the linear arrangement of the proliferation of endothelial cells. EGFL7 possesses the ability to promote the adhesion of endothelial cells, but the ability is weaker than other matrix proteins.

\subsection{Relationship between cancer stem cells and tu- mor angiogenes}

Adult stem cells population is found in normal tissues with capacities of self-renew and multipotent differentiation and its plasticity of adult stem cells is a common phenomenon in organisms. A variety of adult stem cells has been identified, such as skin stem cells, nerve stem cells and adult fat stem cells. Those cells can be differentiated into endothelial progenitor cells or endothelial cells under specific settings. Hypoxia and tissue injury are the initiating events of endothelial differentiation of stem cells. ${ }^{[88]}$ In that way, researchers are accumulating evidence about whether cancer stem cells can differentiate into vascular endothelial cell in tumor angiogenesis. Based on the study results of multi 
energy and embryonic like phenotype of highly aggressive tumors, Bruno and other ${ }^{[89]}$ findings provide direct experimental evidence for the involvement of adult stem cells in tumor angiogenesis through vascular endothelial differentiation. They proved that CD133 + kidney epithelial progenitor cells, which were obtained by separating renal cell carcinoma tissue from normal renal tissues, shared the same biological characteristics with stem cells, without tumorigenicity. CD133 + kidney epithelial progenitor cells showed enhanced ability cause tumor and promote the growth of transplanted tumor enhanced in SCID/NOD mice when combined with $\mathrm{K} 1$ renal carcinoma cell. Xenografts in vivo experiment found that tumor angiogenesis was significantly increased. It is concluded that CD133+ renal epithelial progenitor cells together with co cultured renal cell carcinoma contributes to the formation of vascular endothelial differentiation which serves as an important molecular mechanism for CD133 + kidney epithelial progenitor cells to assist in renal transplantation tumor angiogenesis and accelerate tumor growth. Similar studies in the field of glioblastoma have also been unveiled.

Tumor angiogenesis is the basis of tumor growth and metastasis, varying from the blood vessel in the formation of ovarian corpus, endometrial hyperplasia, wound healing and chronic inflammation. Angiogenesis results from host mature vascular development, while the latter is the result of directional induction and differentiation of endothelial progenitor cells from the bone marrow or circulating system under the stimulation of cytokines in tumor micro environment. ${ }^{[90,91]}$ Additionally, tumor angiogenesis presents in two forms of vessel cooption and vaseulogenic mimicry. Vasculogenic mimicry is more common in tumor tissue with higher aggression, in which endothelial cells are not included, composed of entire tumor cells. ${ }^{[92] ~ V a s c u l o g e n i c ~}$ mimicry and vessels are similar in the structure and function so that it is identified as another circulatory system. ${ }^{[93]}$

No endothelial cells were involved in the lumen-like structure of VM, but the tumor cells were composed of a layer of extracellular matrix, indicating the poor clinical prognosis. The characteristics of VM include: ${ }^{[94]}$ 1) vascular wall is mainly constituted by the tumor cells; 2) no endothelial cells were included in the inner layer of vascular cavity, but a layer of basement membrane-like structures; 3) leakage of red blood cells and micro thrombosis are rare; 4) central necrosis was randomly seen around vasculogenic mimicry; 5) the endothelial specific markers of immune group of [clotting factor VIII (f VIII), Ulex europaeus) Ul-ex and expression of CD31, CD34 and KDR were negatively responded; 6) only appear in highly invasive tumors, other than low invasive tumors or benign tumors. At present, the mechanism of VM is unclear. Past researches mainly focused on genetic characteristics of tumor cells, structural features of vasculogenic mimicry, and tumor microenvironment. The correlation factors which had been reported include VE-cadherin, laminin5, TIE-1,
Eck/A2(EphA2), VEGF-C, MMPs, PI3K, cox-2, TFPI and so on. Malignant cells that constitute vascular mimicry reportedly shared the same features of undifferentiated embryonic stem cell. ${ }^{[95]}$ It is worth noting that anti-tumor angiogenesis would induce the occurrence instead of inhibiting the growth of VM, which may be associated with tumor escape.

Compared with the normal blood vessels, blood vessels of the tumor are immature, and its structures are out of order. It is not easy to distinguish small arteries, veins and capillaries since no normal grading branch presents in tumor blood vessels. Tumor blood vessels are distorted, with uneven surface, uneven wall thickness, irregular diameter size, uneven thickness of Type IV collagen layer on the matrix, and endothelial cells lose normal single molecular layer structure in the arrangement of the vascular wall, and show loose connections to pericytes. The fact that the number and arrangement of peripheral cells were abnormal contributed to the vascular leakage, disturbance of blood flow, higher tissue fluid pressure, and blocking blood flow. Although the tumor tissue is rich in blood vessels, it is often in a state of hypoxia. A very small number of cancer stem cell transplantation leads to high tumor survival rate, but the survival rate of a large number of common tumor cell transplantation is very low. The traditional chemotherapy and other treatment are effective on ordinary tumor cells, but invalid for the tumor stem cells. ${ }^{[96]}$ All these characteristics indicate that the tumor stem cells have a strong proangiogenic function. Tumor stem cells transform into tumor vascular stem / progenitor cells and take part in angiogenesis, or develop into vasculogenie mimicry and got involved in tumor microcirculation. ${ }^{[97-99]}$

\section{Conclusions}

In summary, tumor stem cells can not only differentiate into endothelial cells, but also participate in the regulation of angiogenesis by secreting a variety of angiogenic factors. There is growing acknowledgement that tumor stem cells have similar characteristics with normal stem cells, which provides a new perspective for us to further explore the mechanism of tumor angiogenesis. However, there are still many problems that need to be elucidated. (1) No pecific surface markers are presented in cancer stem cells, and further purification remains challenge. (2) The molecular mechanism of cancer stem cells that involve in tumor occurrence and evolution is unclear. For example, is the rapid growth or reoccurrence of GBM driven by a specific GSCs or all the tumor cells? (3) The difference between molecular mechanisms by which cancer stem cells differentiate into vascular endothelial cells and the molecular mechanisms underlying plasticity of normal tissue stem cells is unclear. For example, the origin of tumor vascular heterogeneity. How do vascular niche and hypoxia niche regulate 
activity of GSCs in space-time. (4) The origin of GSCs. Glioma will be cured thoroughly when all the doubts above are unveiled.

\section{References}

[1] Siegel R, Naishadham D, Jemal A. Cancer statistics, 2012 [J]. CA Cancer J Clin, 2012, 62(1): 10-29.

[2] Fueyo J, Gomez-Manzano C, Yung WK. Advances in translational research in neuro-oncology [J]. Arch Neurol, 2011, 68(3): 303-308.

[3] Carapella CM, Telera S, Oppido PA. Surgery of malignant gliomas: advances and perspectives [J]. Curr Opin Oncol, 2011, 23(6): 624629.

[4] Reya T, Morrison SJ, Clarke MF, et al. Stem cells, cancer, and cancer stem cells [J]. Nature, 2001, 414(6859): 105-111.

[5] Singh S K, Hawkins C, Clarke I D, et al. Identification of human brain tumour initiating cells [J]. Nature, 2004, 432: 396-401.

[6] Dick JE. Looking ahead in cancer stem cell research [J]. Nat Biotechnol, 2009, 27(1): 44-46.

[7] Dean M, Fojo T, Bates S. Tumour stem cells and drug resistance [J]. Nat Rev Cancer, 2005, 5(4): 275-284.

[8] Clarke MF, Dick JE, Dirks PB, et al. Cancer stem cells-perspectives on current status and future directions: AACR Workshop on cancer stem cells [J]. Cancer Res, 2006, 66(19): 9339-44.

[9] Lapidot T, Sirard C, Vormoor J, et al. A cell initiating human acute myeloid leukaemia after transplantation into SCID mice [J]. Nature, 1994, 367(6464): 645-648.

[10] Bonnet D, Dick J E. Human acute myeloid leukemia is organized as a hierarchy that originates from a primitive hematopoietic cell [J]. Nature Med, 1997, 3(7): 730-737.

[11] Sutherland H J, Blair A, Zapf R W. Characterization of a hierarchy in human myeloid leukemia progenitor cells [J]. Blood, 1996, 87(11): 4754-4761.

[12] Dick JE. Stem cell concepts renew cancer research [J]. Blood, 2008, 112(13): 4793-4807.

[13] Al-Hajj M, Clarke M F. Self-renewal and solid tumor stem cells [J]. Oncogene, 2004, 23(43): 7274-7282.

[14] Qiang Huang. Glioma cells and their role in the genesis of gliomas [J]. Chinese Journal of Neurosurgery, 2006, 22(12): 773-774.

[15] Takahashi K, Yamanaka S. Induction of pluripoten stem cells from mouse embryonic and adult fibroblast cultures by defined factors [J]. Cell, 2006, 126(4): 663-676.

[16] Liang Y, Zhong Z, Huang Y, et al. Stem-like cancer cells are inducible by increasing genomic instability in cancer cells [J]. J Biol Chem, 2010, 285(7): 4931-4940.

[17] Chen Li, Shen R, Ye Y, et al. Precancerous stem cells have the potential for both benign and malignant differentiation [J]. PLoS One, 2007, 2(3): e293.

[18] Hu M, Polyak K. Microenvironmental regulation of cancer development [J]. Curr Opin Genet Dev, 2008, 18(1): 27-34.

[19] Su X, Ye J, Hsueh EC, et al. Tumor microenvironments direct the recruitment and expansion of human Th17cells [J]. J Immunol, 2010, 184(3): 1630-1641.

[20] Cho RW, Clarke MF. Recent advances in cancer stem cells [J]. Curr Opin Genet Dev, 2008, 18(1): 48-53.

[21] Kemper K,Sprick MR,de Bree M,et al.The AC133 epitope, but not the CD133 protein is lost upon cancer stem cell differentiation [J]. Cancer Res, 2010, 70(2): 719-729.

[22] Beier D, Hau P, Proescholdt M, et al. CD133(+)and CD133(-) glioblastoma-derived cancer stem cells show differential growth characteristics and molecular profiles [J]. Cancer Res, 2007, 67(9): 4010-4015.

[23] Günther HS, Schmidt NO, Phillips HS, et al. Glioblastoma-derived stem cell-enriched cultures form distinct subgroups according to molecular and phenotypic criteria [J]. Oncogene, 2008, 27(20): 2897-2909.

\section{Conflicts of Interest Disclosure}

The authors have no conflict of interest related to this article.

[24] Wang J, Sakariassen P, Tsinkalovsky O, et al. CD133 negative glioma cells form tumors in nude rats and give rise to CD133 positive cells [J]. Int J Cancer, 2008, 122(4): 761-768.

[25] Joo KM, Kim SY, Jin X, et al.Clinical and biological implications of CD133-positive and CD133-negative cells in glioblastomas [J]. Lab Invest, 2008, 88(8): 808-815.

[26] Cho DY, Lin SZ, Yang WK, et al. The Role of Cancer Stem Cells (CD133+) in Malignant Gliomas [J]. Cell Transplant, 2011, 20(1): 121-125.

[27] Zhang SJ, Ye F, Xie RF, et al. Comparative study on the stem cell phenotypes of $\mathrm{C} 6$ cells under different culture conditions $[\mathrm{J}]$. Chin Med J(Engl), 2011, 124(19): 3118-3126.

[28] Lottaz C, Beier D, Meyer K, et al. Transcriptional profiles of CD133+ and CD133- glioblastoma-derived cancer stem cell lines suggest different cells of origin [J]. Cancer Res, 2010, 70(5): 20302040.

[29] Tchoghandjian A, Baeza N, Colin C, et al. A2B5 cells fromhuman glioblastoma have cancer stem cell properties [J]. BrainPathol, 2010, 20(1): 211-221.

[30] Ogden AT, Waziri AE, Lochhead R, et al. Dentification of A2B5+ CD133- tumor-initiating cells in adult human gliomas [J].Neurosurgery, 2008, 62(2): 505-514.

[31] Christensen K, Aaberg-Jessen C, Andersen C, et al. Immunohistochemical expression of stem cell, endothelial cell, and chemo sensitivity markers in primary glioma spheroids cultured in serumcontaining and serum-free medium [J]. Neurosurgery, 2010, 66(5): 933-947.

[32] Annovazzi L, Mellai M, Caldera V, et al.SOX2 expression and amplification in gliomas and glioma cell lines [J].Cancer Genomics Proteomics, 2011, 8(3): 139-14.

[33] Broadley KW, Hunn MK, Farrand KJ, et al. Side Population is Not Necessary or Sufficient for a Cancer Stem Cell Phenotype in Glioblastoma Multi forme [J].Stem Cells, 2011, 299(3): 452-461.

[34] Hendrix M J, Seftor E A, Seftor R E, et al. Reprogramming metastatic tumour cells with embryonic microenvironments [J].Nat Rev Cancer, 2007, 7: 246-255.

[35] Mitsiadis T A, Barrandon O, Rochat A, et al. Stem cell niches in mammals [J]. Exp Cell Res, 2007, 313: 3377-3385.

[36] Chen R, Nishimura MC, Bumbaca SM, et al. A hierarchy of selfrenewing tumor-initiating cell types in glioblastoma [J].Cancer Cell, 2010, 17(4): 362-375.

[37] Liotta LA, Kohn EC. The microenvironment of the tumour-host interface [J]. Nature, 2001, 17; 411(6835): 375-379.

[38] Denysenko T, Gennero L, Roos MA, et al. Glioblastoma cancer stem cells: heterogeneity, microenvironment and related therapeutic strategies [J]. Cell Biochem Funct, 2010, 28(5): 343-351.

[39] Voss MJ, Niggemann B, Zänker KS, et al. Tumour reactions to hypoxia [J]. Curr Mol Med, 2010, 10(4): 381-386.

[40] Zhu P, Ning Y, Yao L,et al. The proliferation, apoptosis, invasion of endothelial-like epithelial ovarian cancer cells induced by hypoxia. J Exp Clin Cancer Res, 2010, 29(1): 124-131.

[41] John M. Heddleston, Zhizhong Li,et al. The Hypoxic Microenvironment Maintains Glioblastoma Stem Cells and Promotes Reprogramming towards a Cancer Stem Cell Phenotype [J]. Cell Cycle, 2009, 15; 8(20): 3274-3284.

[42] de Almeida Sassi F, Lunardi Brunetto A, Schwartsmann G, et al. Glioma Revisited: From Neurogenesis and Cancer Stem Cells to the Epigenetic Regulation of the Niche [J]. J Oncol, 2012, 2012: 537861.

[43] Kim Y, Lin Q,Glazer PM, et al. Hypoxic tumor microenvironment and cancer cell differentiation [J]. Curr Mol Med, 2009, 9: 425-434. 
[44] Fraga A, Ribeiro R, Medeiros R. Tumor hypoxia: the role of HIF [J]. Actas Urol Esp, 2009, 33(9): 941-951.

[45] Vaupel P, Mayer A. Hypoxia in cancer: significance and impact on clinical outcome [J]. Cancer Metastasis Rev, 2007, 26(2): 225-239.

[46] Jianchun Zong, Chengyi Wu. VEGF expression in human breast carcinoma cell line induced by hypoxia and its mechanism [J]. Journal of Chongqing Medical University, 2005, 30(2): 206-209.

[47] Erkan M, Reiser-Erkan C, Michalski CW, et al. Cancer stellate cell interactions perpetuate the hypoxia-fibrosis cycle in pancreatic ductal adenocarcinoma [J]. Neoplasia, 2009, 11(5): 497-508.

[48] Acker T, Diez-Juan A, Aragones J, et al. Genetic evidence for a tumor suppressor role of HIF-2alpha [J]. Cancer Cell, 2005, 8(2):131141.

[49] Li Z, Bao S, Wu Q, et al. Hypoxia-inducible factors regulate tumorigenic capacity of glioma stem cells [J]. Cancer Cell. 2009, 15(6): 501-513.

[50] Pietras A, Hansford LM, Johnsson AS, et al. HIF-2alpha maintains an undifferentiated state in neural crest-like human neuroblastoma tumor initiating cells [J]. Proc Natl Acad Sci U S A, 2009, 106(39): 16805-16810.

[51] Pietras A, Gisselsson D, Ora I, et al. High levels of HIF-2alpha highlight an immature neural crest-like neuroblastoma cell cohort located in a perivascular niche [J]. J Pathol, 2008, 214(4): 482-488.

[52] Seidel S, Garvalov BK, Wirta V,et al. A hypoxic niche regulates glioblastoma stem cells through hypoxia inducible factor 2 alpha [J]. Brain, 2010, 133(Pt4): 983-995.

[53] Calabrese C, Poppleton H, Kocak M,et al. A perivascular niche for brain tumor stem cells [J]. Cancer Cell, 2007, 11(1): 69-82.

[54] Hambardzumyan D, Becher OJ, Rosenblum MK, et al. PI3K pathway regulates survival of cancer stem cells residing in the perivascular niche following radiation in medulloblastoma in vivo [J]. Genes Dev, 2008, 22(4): 436-448.

[55] Liotta LA, Kohn EC, Petricoin EF. The microenvironment of the tumour-host interface [J]. Nature, 2001, 411 (17): 375-378.

[56] Melillo G, Semenza GL. Meeting report: exploiting the tumor microenvironment for therapeutics [J].Cancer Res, 2006, 66(9): 45584560.

[57] Witz IP. Tumor-microenvironment interactions: the selectin-selectin ligand axis in tumor-endothelium cross talk [J]. Cancer Treat Res, 2006, 130(25): 125-140

[58] Hall B, Dembinski J, Sasser AK, et al. Mesenchymal stem cells in cancer: tumor-associated fibroblasts and cell-based delivery vehicles [J]. Int J Hematol, 2007, 86(1): 8-16.

[59] Ghebeh H, Dermime S. Comment on "Characterization of human lung tumor- associated fibroblasts and their ability to modulate the activation of tumor- associated T cells"[J]. J Immunol, 2007, 179(2): $732,733$.

[60] Henry LR, Lee HO, Lee JS, et al. Clinical implications of fibroblast activation protein in patients with colon cancer [J]. Clin Cancer Res, 2007, 13(6): 1736-1741.

[61] Lamagna C, Aurrand-Lions M, Imhof BA. Dual role of macrophages in tumor growth and angiogenesis [J]. J Leukoc Biol, 2006, 80(4): 705-713.

[62] Luo Y, Zhou H, Krueger J, et al. Targeting tumor-associated macrophages as a novel strategy against breast cancer [J]. J Clin Invest, 2006, 116(8): 2132-2141.

[63] Bussolati B, Grange C, Tei L, et al. Targeting of human renal tumorderived endothelial cells with peptides obtained by phage display [J]. J Mol Med, 2007, 85(8): 897-906.

[64] Lu C, Bonome T, Li Y, et al. Gene alterations identified by expression profiling in tumor-associated endothelial cells from invasive ovarian carcinoma [J]. Cancer Res, 2007, 67(4): 1757-1768.

[65] L Addison C. Modulation of response to tumor therapies by the extracellular matrix [J]. Future Oncol, 2006, 2(3): 417-429.

[66] Howell GM, Grandis JR. Molecular mediators of metastasis in head and neck squamous cell carcinoma [J]. Head Neck, 2005, 27(8): 710-717.

[67] Bonfil RD, Chinni S, Fridman R, et al. Proteases, growth factors, chemokines, and the microenvironment in prostate cancer bone metastasis [J]. Urol Oncol, 2007, 25(5): 407-411.
[68] Boeuf L, Houle F, Huot F, et al. Regulation of vascular endothelial growth factor receptor 2-mediated phosphorylation of focal adhesion kinase by heat shock protein 90 and Src kinase activities [J]. Journal of Biological Chemistry, 2004, 279(37): 39175-39185.

[69] Gajewski TF. Failure at the effector phase: immune barriers at the level of the melanoma tumor microenvironment $[\mathrm{J}]$. Clin Cancer Res, 2007, 13(18): 5256- 5261.

[70] Khosravi Shahi P, Fernández Pineda I. Tumoral angiogenesis: review of the literature [J]. Cancer Invest, 2008, 26(1): 104-108.

[71] Folkman J. Tumor angiogenesis: therapeutic implications. N Engl J Med, 1971, 285(21): 1182-1186.

[72] Ratcliffe PJ, Pugh CW, Maxwell PH. Targeting tumors through the HIF system. [J]. Nat Med, 2000, 6(12): 1315-1316.

[73] Folkins C, Man S, Xu P, et al. Anticancer therapies combining antiangiogenic and tumor cell cytotoxic effects reduce the tumor stemlike cell fraction in glioma xenograft tumors. Cancer Res, 2007, 67(8): 3560-3564.

[74] Veeravagu A, Hsu AR, Cai W,et al. Vascular endothelial growth factor and vascular endothelial growth factor receptor inhibitors as antiangiogenic agents in cancer therapy [J]. Recent Pat Anticancer Drug Discov, 2007, 2(1): 59-71.

[75] Bulnes S, Lafuente JV. VEGF immunopositivity related to malignancy degree, proliferative activity and angiogenesis in ENUinduced gliomas [J]. J Mol Neurosci, 2007, 33(2): 163-172.

[76] Karcher S, Steiner HH, Ahmadi R,et al. Different angiogenic phenotypes in primary and secondary glioblastomas [J]. Int J Cancer, 2006, 118(9): 2182-2189.

[77] Brekken RA, Overholser JP, Stastny VA, et al. Selective inhibition of vascula endothelial growth factor (VEGF) receptor 2(KDR/FIk1) activity by a monoclona antiVEGF antibody blocks tumor growth in mice. Cancer Res, 2000, 60(18): 5117-5124.

[78] Li G, Chen Z, Hu YD, et al. Autocrine factors sustain glioblastoma stem cell self-renewal. Oncol Rep, 2009, 21(2): 419-424.

[79] Park DM, Rich JN. Biology of glioma cancer stem cells. Mol Cells, 2009, 28(1): 7-12.

[80] de la Torre NG, Buley I, Wass JA,et al. Angiogenesis and lymphangiogenesis in thyroid proliferative lesions: relationship to type and tumour behaviour. Endocr Relat Cancer, 2006, 13(3): 931-44.

[81] El-Assal ON, Yamanoi A, Ono T,et al. The clinicopathological significance of heparanase and basic fibroblast growth factor expressions in hepatocellular carcinoma [J]. Clin Cancer Res, 2001, 7(5): 1299-305.

[82] Nomi M, Miyake H, Sugita Y, et al. Role of growth factors and endothelial cells in therapeutic angiogenesis and tissue engineering [J]. Curr Stem Cell Res Ther, 2006, 1(3): 333-43.

[83] Ganter MT, Cohen MJ, Brohi K, et al. Angiopoietin-2, marker and mediator of endothelial activation with prognostic signnificance early after trauma? [J]. Ann Surg, 2008, 247(2): 320-326.

[84] Lee OH, Xu J, Fueyo J,et al. Angiopoietin-2 decreases vascular endothelial growth factor expression by modulating HIF-1 alpha levels in gliomas [J]. Oncogene, 2008, 27(9): 1310-1314.

[85] Hu B, Jarzynka MJ, Guo P, et al. Angiopoietin 2 induces glioma cell invasion by stimulating matrix metalloprotease 2 expression through the alphavbetal integrin and focal adhesion kinase signaling pathway [J]. Cancer Res, 2006, 66(2): 775-783.

[86] Fitch MJ, Campagnolo L, Kuhnert F,et al. Egfl7, a novel epidermal growth factor- domain gene expressed in endothelial cells [J]. Dev Dyn, 2004, 230(2): 316-324.

[87] Campagnolo L, Leahy A, Chitnis S,et al. EGFL7 is a chemoattractant for endothelial cells and is up-regulated in angiogenesis and arterial injury [J]. Am J Pathol, 2005, 167(1): 275-284.

[88] Li Z, Bao S, Wu Q, et al. Hypoxia-inducible factors regulate tumorigenic capacity of glioma stem cells [J]. Cancer Cell, 2009, 15(6): 501- 513.

[89] Bruno S, Bussolati B, Grange C, et a1. CD133+ renal progenitor cells contribute to tumor angiogenesis [J]. Am J Pathol, 2006, 169(6): 2223-2235.

[90] Hillen F, Griffioen AW. Tumour vascularization: sprouting angiogenesis and beyond [J].Cancer Metastasis Rev, 2007, 26(3-4): 489502. 
[91] Folkman J, Beckner K. Angiogenesis imaging [J]. Acad Radiol, 2000, 7(10): 783-785.

[92] Hillen F, Griffioen AW. Tumour vascularization: sprouting angiogenesis and beyond [J]. Cancer Metastasis Rev, 2007, 26(3-4): 489502.

[93] Folberg R, Hendrix MJ, Maniotis AJ. Vasculogenic mimicry and tumor angiogenesis [J]. Am J Pathol, 2000, 156(2): 361-381.

[94] Sun B, Zhang D, Zhang S, et al. Hypoxia influences vasculogenic mimicry channel formation and tumor invasion-related protein expression in melanoma [J]. Cancer Lett, 2007, 249(2): 188-197.

[95] Hendrix MJ, Seftor EA, Hess AR, et al. Vasculogenic mimicry and tumour-cell plasticity: lessons from melanoma. Nat Rev Cancer.
2003, 3(6):411-421.

[96] Shervington A, Lu C. Expression of multidrug resistance genes in normal and cancer stem cells. Cancer Invest, 2008, 26(5): 535-542.

[97] Bao S, Wu Q, Sathornsumetee S, et al. Stem cell-like glioma cells promote tumor angiogenesis through vascular endothelial growth factor. Cancer Res, 2006, 66(16): 7843-7848.

[98] Wang R, Chadalavada K, Wilshire J, et al. Glioblastoma stem-like cells give rise to tumour endothelium. Nature, 2010, 468(7325): 829-833.

[99] Ricci-Vitiani L, Pallini R, Biffoni M, et al. Tumour vascularization via endothelial differentiation of glioblastoma stem-like cells. Nature, 2010, 468: 824-8. 